\title{
VR-EA: Virtual Reality Visualization of Enterprise Architecture Models with ArchiMate and BPMN
}

\author{
Roy Oberhauser ${ }^{10000-0002-7606-8226]}$ and Camil Pogolski ${ }^{1}$ \\ Computer Science Dept., Aalen University, Aalen, Germany \\ ${ }^{1}$ \{roy.oberhauser, camil.pogolski\}@hs-aalen.de
}

\begin{abstract}
The digital transformation occurring throughout enterprises results in an increasingly dynamic and complex IT landscape. As the structures with which enterprise architecture (EA) deals become more digital, larger, complex, and dynamic, new approaches for modeling, documenting, and conveying EA structural and relational aspects are needed. The potential for virtual reality (VR) to address upcoming EA modeling challenges has as yet been insufficiently explored. This paper contributes a VR hypermodel solution concept for visualizing, navigating, interacting with ArchiMate and Business Process Modeling Notation (BPMN) models in VR. An implementation demonstrates its feasibility and a case study is used to show its potential.
\end{abstract}

Keywords: Virtual Reality, Enterprise Architecture, Business Process Models, BPMN, ArchiMate, Visualization.

\section{Introduction}

Multiple contemporaneous trends and challenges are being faced by enterprises, including the smaller and not so information technology (IT)-centric enterprises: 1) digital transformation/automation, 2) agile business processes, and 3) servicenetworked software with frequent deployments. First, a major digital transformation of industries is underway (Muro et al., 2017). While the digitalization rate (digital score) may vary across industries and economies, it is nevertheless impacting business strategies and necessarily the enterprise architecture (EA) that supports the business. As big data, data analytics, business intelligence, and machine learning make inroads into enterprises, improved decision-making capabilities at all levels and across organizational entities empowers employees with new insights and assistance and bringing further automation that in turn affects business functions and processes, and thus affecting and changing the actual EA. Second, enterprises are facing competitive pressure to implement agile business processes that are fast, lean, and more effective, with enterprise management, processes, and projects moving towards continuously flexible, responsive, and efficient business forms to accelerate product and service delivery (e.g., Scrum, DevOps, BizDevOps). The IT infrastructure needed to support these dynamic processes implies a much more dynamic and complex EA to support these both rapidly changing and highly-integrated business processes. Finally, 
the IT infrastructure is rapidly changing from local, siloed, and static deployments to cloud-centric, networked, and containerized micro-functionality with frequent new deployments (Forsgren et al., 2017). Software/data functionality becomes easily reusable and accessible via standard protocols and formats independent of programming language or platform, and thus more connected and integrated. The scale and impact on IT landscapes can be appreciated via various "death star"-like microservice network landscape visualizations (Munns, 2015) as exemplified in Fig. 1.

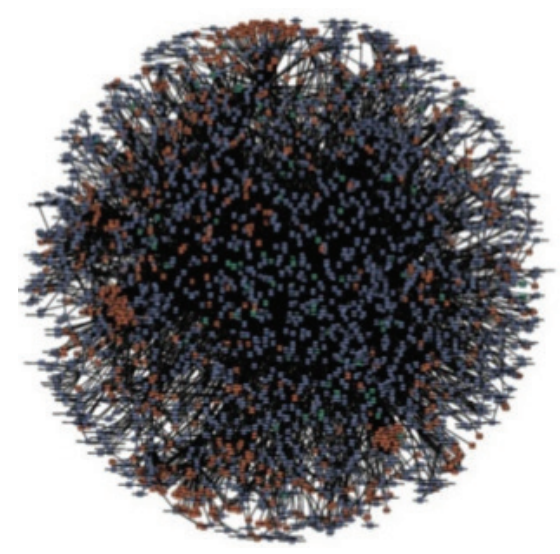

Fig. 1. Visualization of microservices at Amazon (Munns, 2015)

Enterprise architecture (EA) comprises the structural and behavioral aspects needed for an enterprise to function and adapt in alignment with some vision. To this end, it involves comprehensive and cohesive modeling and documentation. Considering the trends and challenges mentioned, the reality that EA is attempting to comprehensively model, document, and change has become much more complex than in previous decades. To cope with this new reality, new EA modeling paradigms and EA modeling tool approaches are needed. EA seeks to provide a comprehensive set of cohesive models to describe the enterprise structure and functions, while individual models are logically arranged to provide further detail about an enterprise (Jarvis, 2003).

Virtual Reality (VR) could potentially assist with visualizing this growing and complex set of models and their interrelationships. VR is defined as a "real or simulated environment in which the perceiver experiences telepresence" (Steuer, 1992), a mediated visual environment which is created and then experienced. VR has made inroads in various domains and become readily accessible as hardware prices have dropped and capabilities improved. As EA models grow in complexity and reflect the deeper integration of both the business and IT reality, an immersive EA environment could provide an additional visualization capability to comprehend the "big picture" for structurally and hierarchically complex and interconnected diagrams, while providing an immersive experience for EA models in a 3D space viewable from different perspectives. 
As to modeling EA, ArchiMate (Open Group, 2017) provides a visual EA modeling language for visualizing, describing, and analyzing the various elements involved in EA. This includes business and organizational structures, business processes, information flows, IT applications and systems, and technical infrastructure. While the scope of ArchiMate is used to model high-level enterprise processes and to depict their relations to the enterprise context, it is not intended to be a detailed workflow modeling language. For this, the Business Process Model and Notation (BPMN) (OMG, 2011) supports the modeling of detailed subprocesses and tasks from the abstract to an executable specification. However, BPMN does not cope with modeling the wider enterprise context like the goals and requirements the process is intended to fulfill, nor for modeling the supporting application services and infrastructure. Thus, the two notations can be viewed as complementing one another in providing more context for a business process, while providing sufficient detail of a business process to the point of executability. In our view, various parts of the detailed business process could and should be seen within the larger enterprise scope to better understand the business process context and any potential dependencies.

In general, modeling provides an abstracted or simplified representation of a system that can assist with understanding relationships between elements or concepts of interest. Typically, views are used to represent some stakeholder concern and portray the relevant aspects of the model. However, with typical 2D view depictions, one can lose insight into the interrelationships across views and where relevant model elements are to be found. By leveraging the third dimension provided in VR, the overall interrelationships of the models and views can be indicated and considered.

In prior work, we described VR-BPMN (Oberhauser et al., 2018), our VR solution concept for visualizing, navigating, annotating, and interacting with BPMN models that supports teleportation, fly -through navigation, depicting subprocesses via stacked hyperplanes, drawing annotative associations between BPMN elements, coloring model elements, and tagging textual elements using mixed reality keyboard support. That approach and implementation resulted in a BPMN-specific VR solution. For this paper, we have generalized our approach to enable visualization of generic model elements, rather than many specific shapes, we use cubes with a textual label and optionally displaying any assigned visual icon to represent the element type. Relationships between elements can be shown, and related elements can be grouped in layers or views. We applied this generalized approach to leverage an immersive VR capability for EA and BPMN models. This paper contributes a generalized VR-MF and the more specific VR-EA, a VR hypermodel solution concept for EA model visualization, navigation, and interaction. To address the deeper integration, complexity, and interconnectedness of EA with business processes, it facilitates the convergence of Archimate enterprise models and BPMN models in VR, enabling both to be visualized and analyzed in the same field of view. Details on the implementation prototype are provided, and we evaluate our implementation using the ArchiSurance case study.

The remainder of this paper is structured as follows: Section 2 discusses related work. In Section 3 the VR-EA solution concept is described. Section 4 then provides details on our prototype implementation. The evaluation using a case study is described in Section 5, and a conclusion follows in Section 6. 


\section{Related Work}

Work related to EA visualization includes (Rehring et al., 2019), who applied 3D visualization in augmented reality in support of EA decision making. (Naranjo et al., 2014) describe PRIMate based on PRIMROSe, a visual graph-based enterprise analysis framework, and show a graph, treemap, and 3D visualization of an the ArchiSurance ArchiMate model. As to harmonizing ArchiMate, BPMN, and UML, (van den Berg, 2012) analyzes the various metamodels and shows how one could practically combine the notations across views and diagrams. We are unaware of any VRspecific work with ArchiMate, EA model convergence, or EA hypermodels.

BPMN-related work includes the process visualization and virtualization areas. As to process visualization techniques, (Du, Gu, \& Zhu, 2012) provide a survey, concluding that $3 \mathrm{D}$ can improve the layout and can increase the information content of process models. Work related to process visualization includes (Betz et al., 2008), who described an approach for 3D representation of business process models based on Petri nets with organizational models, showing that a $3 \mathrm{D}$ process representation can facilitate process-specific information access. (Hipp et al., 2014) described and empirically evaluated various business process visualization alternatives: bubbles, BPMN3D that maps data objects associated to a BPMN element to a "third dimension", network, and thin lines; however, no 3D space nor an implementation is described. With regard to virtual worlds, (Brown, Recker, \& West, 2011) investigated collaborative process modeling and communication, implementing a 3D BPMN modeling environment in the virtual world Second Life, and also used the Open Simulator (Brown, 2010). The 3D Flight Navigator (Effinger, 2012) was implemented in Java with OpenGL, and projects parts of BPMN collaboration diagrams onto fixed hyperplanes and provides a heads-up display for navigating the process. No major BPMS vendors currently sell VR variants of their products.

In contrast, out VR-MF/VR-EA solution concept enables VR-centric visualization, can be implemented on standard game engine technology (Unity) and uses common VR hardware (HTC Vive). It supports hypermodeling, e.g., combining ArchiMate and BPMN models in the same space, provides automatic layout of views as stacked 3D hyperplanes, and visualizes the reality of inter-view relations of elements.

\section{Solution Concept}

Our generalized VR modeling solution concept provides a VR-based domainindependent hypermodeling framework (VR-MF shown in Fig. 2) that addresses three primary aspects especially affecting models in VR: visualization, navigation, and interaction. Rather than requiring unique and specific 3D shapes, we use cubes with a textual label and display an optional type icon (if provided) on the cube sides. Relationships between elements can be shown, and related elements can be grouped in layers or views. Our support for EA models in VR is called VR-EA in Fig. 2, and encompasses both BPMN and ArchiMate models. 


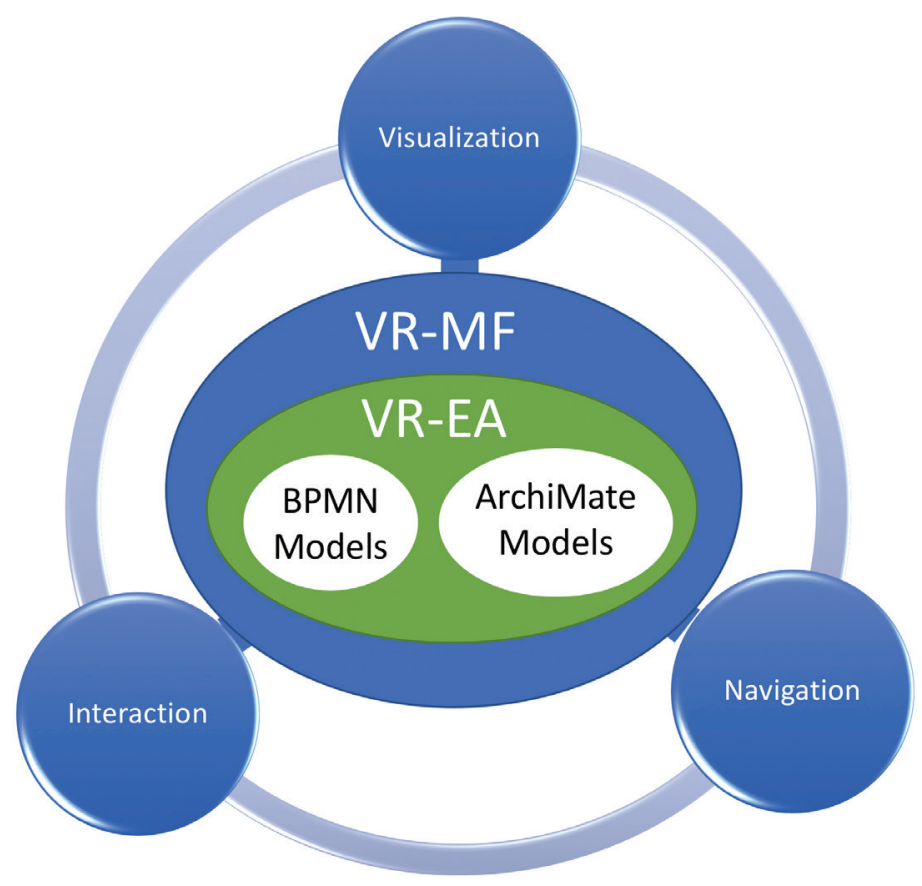

Fig. 2. The VR-MF (general) and VR-EA (EA-specific) solution concept

ArchiMate models use a graphical notation consisting of a collection of concepts (approximately 50) to portray a wide scope of EA elements and relationships. Elements can be behavioral, structural, motivational, or some composite. These concepts can participate in various layers: strategy, business, application, technology, physical, and implementation \& migration, the layers having colors associated with them. Cross-cutting aspects involved include: passive structure, active structure, behavior, and motivation. Views are used to convey information addressing concerns of specific stakeholders. On the other hand, BPMN models focus on business processes and consist of Business Process Diagrams (BPDs) composed of graphical elements consisting of flow objects, connecting objects, swim lanes, and artifacts (OMG, 2011).

Visualization. The graphical elements involved in ArchiMate and BPMN are specified in 2D. While many visual options and metaphors are possible, our view is that diverging too far from the images in the specification would reduce the recognition and standardization afforded by the respective specification. Thus, to differentiate elements by type, we chose to use generic 3D cubes and project the relevant standard 2D image for the object type onto all sides as a texture, which can thus be perceived from all angles. In contrast to 2D space, one challenge in 3D space element placement is that one can never be sure if an element is not hidden behind another element at any particular vantage point if the element is opaque. However, if one makes the element partially transparent, then it can become confusing as to which element one is actually focusing on. We thus chose to make the elements opaque in order to avoid this visual 
confusion, and by briefly adjusting one's perspective one can visually check that nothing is hidden behind an element. Moreover, visualizing text is an issue in VR due to the relatively low resolutions currently available and the distance from the virtual camera position to the text. Also, labels for ArchiMate and BPMN elements can differ widely in length, yet should not interfere with understanding the underlying diagram structure. We thus place labels above the elements (analogous to billboards), make them partially transparent in order not to completely hide any elements behind the label, and the labels are made to automatically rotate towards the camera to improve legibility from any given angle. For dealing with longer labels we constrain the maximum billboard width (to reduce over-lapping), raise the billboard height, and reduce the text font size. For visualizing and differentiating the various diagrams, hyperplanes are used to take advantage of the 3D space, with each plane representing one diagram. In BPMN, subprocesses are projected onto a plane beneath its superprocess.

The ArchiMate specification advises “...viewpoints and views...should not be considered in isolation: views are inter-related and, often, it is exactly a combination of views together with their underlying inter-dependency relationships that is the best way to describe and communicate a piece of architecture. It should, however, be noted that viewpoints and views have a limiting character. They are eventually a restriction of the whole system (and architecture) to a partial number of aspects - a view is just a partial incomplete depiction of the system" (Open Group, 2017). Consequently, our VR-EA solution involves a reality-centric principle for visualization. What we mean by this is that by default we depict the true complexity and interrelatedness of the model element, permitting the user to hide the views, layers, or elements that are currently not of interest. View-centric portrayals be definition necessarily hide these aspects and thus parts of this reality. Given the growing complexity in EA and IT landscapes, we believe an over-simplification can be detrimental to understanding, e.g. by potentially overlooking a key dependency across views or requiring a user to remember to look at multiple views to see the overall impact of a change. While current ArchiMate tools such as Archi provide an automatic Visualiser or View Generator, they are limited to the single element in focus.

Navigation. The immersion afforded by VR requires addressing how to intuitively navigate the space while reducing the likelihood of potential VR sickness symptoms. Two navigation modes are included in the solution concept: the default uses gliding controls, enabling users to fly through the VR space and get an overview of the entire model from any angle they wish. Alternatively, teleporting permits a user to select a destination and be instantly placed there (i.e., by moving the camera to that position); this can be disconcerting but may reduce the likelihood of VR sickness that can occur when moving through a virtual space for those prone to it.

Interaction. Neither ArchiMate nor BPMN specify exactly how users are to interact and interface with graphical visual elements. In our VR concept, user-element interaction is done primarily via the VR controllers. Views are stacked hyperplanes and can be made visible or invisible by selecting the plane. Inter-view connections can be enabled or disabled. A specific connection can be selected to emphasize it. 


\section{$4 \quad$ Realization}

Initially, our realization primarily addresses visualization and navigation of existing models. Future work will include support for creating and improving models in VR.

The Unity game engine was chosen for VR visualization due to its multi-platform support, direct VR integration, popularity, and cost, and we utilize the SteamVR plugin and runtime. Blender was used to develop certain visual model elements. For testing with VR hardware, we used the HTC Vive, a room scale VR set with a headmounted display and two wireless handheld controllers tracked by two base stations.

The implementation architecture for our prototype is shown in Fig. 3. The Unity engine makes use of the various assets such as Models, Scenes, and Scripts, which in turn access external model files. A plugin adapter interface allows various model file formats (e.g., BPMN, ArchiMate) to be parsed and converted to the internal generic object representation.

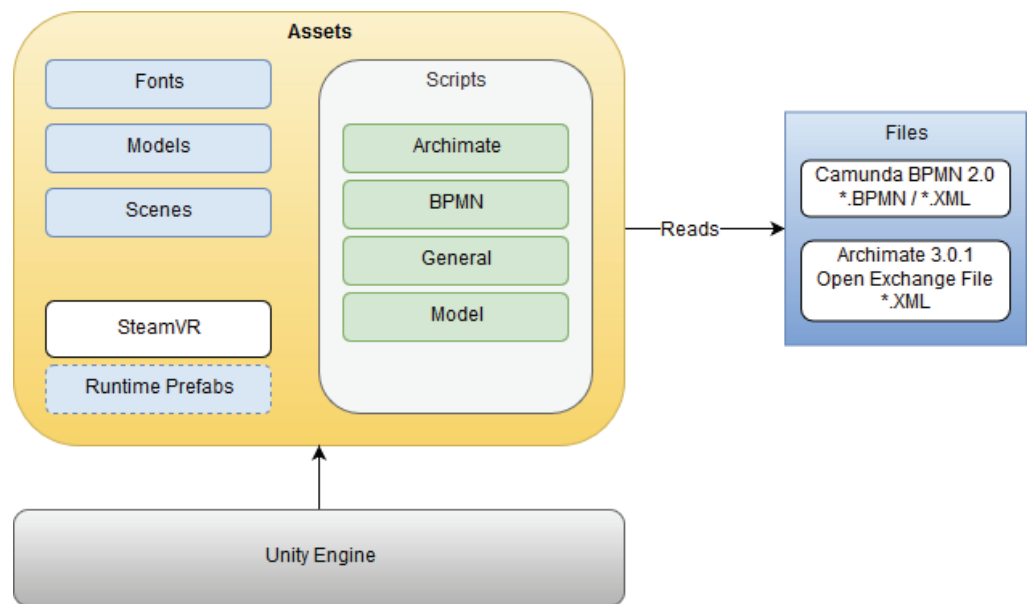

Fig. 3. VR-EA implementation architecture

Generic cubes are used to depict model elements. If an icon is assigned to the type, it is depicted on all 6 sides, otherwise the element type (as text) is shown instead (see Fig. 4 where BusinessService is projected on the Claims Payment Service cube). The positioning of elements within a view or layer is done by reading out the position in the models and applying an adjustable scaling factor (0.05 for BPMN and 0.04 for ArchiMate). Ordering of the views as stacked layers is done relative to each other to avoid collisions and to better portray relations and cluster by known views. The hypermodeling capability is shown in Fig. 5, where two BPMN models (an Invoice Process (left) (ORYX-Editor, 2009) and Insurance Claim Process (center) (GitHub, 2017)) are shown in the same layer (vertical height) as the Business Process View from the ArchiMate model (based on ArchiSurance (Open Group, 2017a)). For comparison, Fig. 6 shows the BPMN Insurance Claim Processing model from Fig. 5 as the typical non-VR BPMN diagram. 


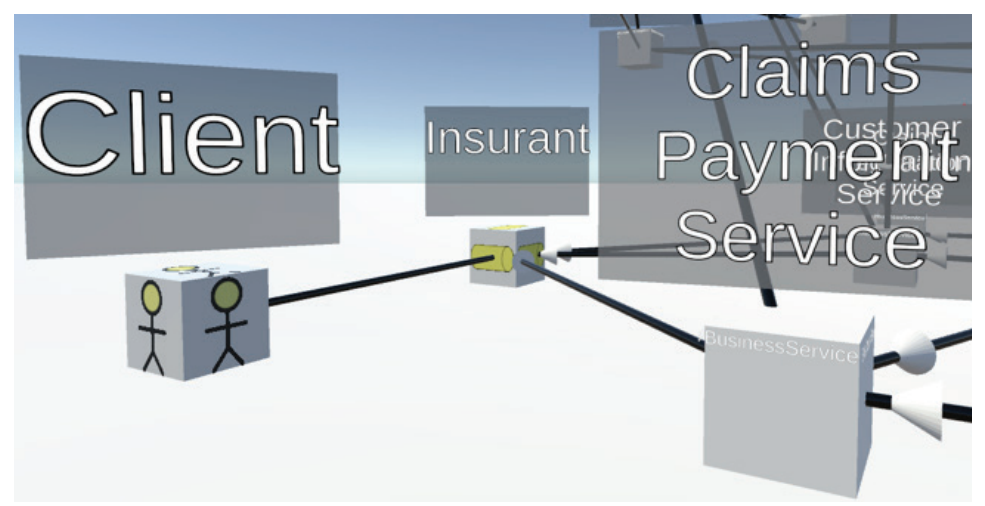

Fig. 4. VR-EA cubes with and without icons (labeled with the element type if unassigned)

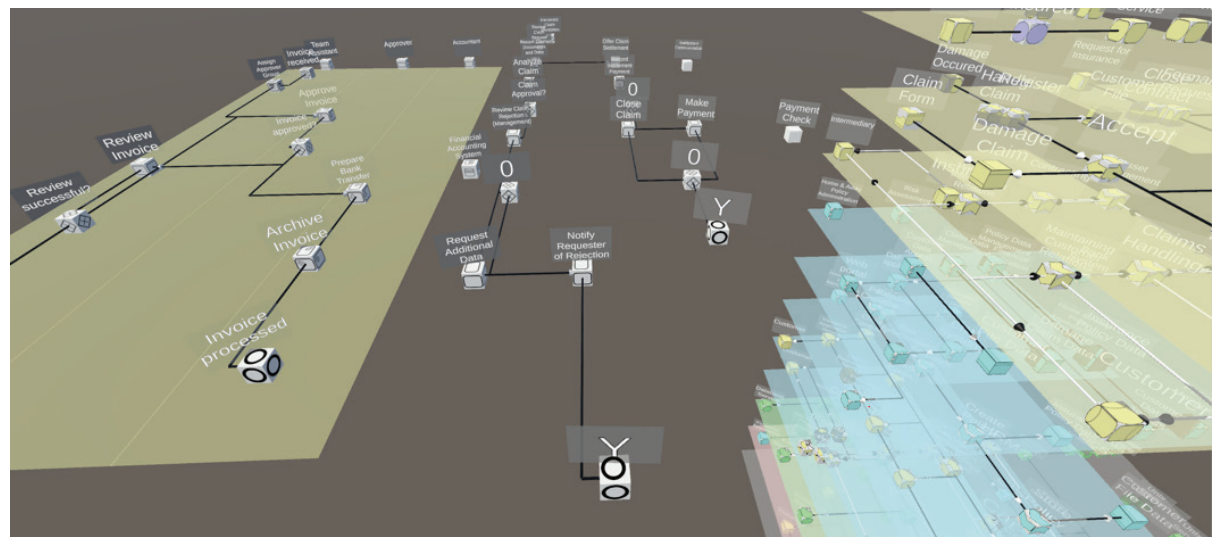

Fig. 5. VR-EA hypermodel showing BPMN models (left and center) in the same vertical layer as the Business Process View in the ArchiMate model (ArchiSurance) on the right.

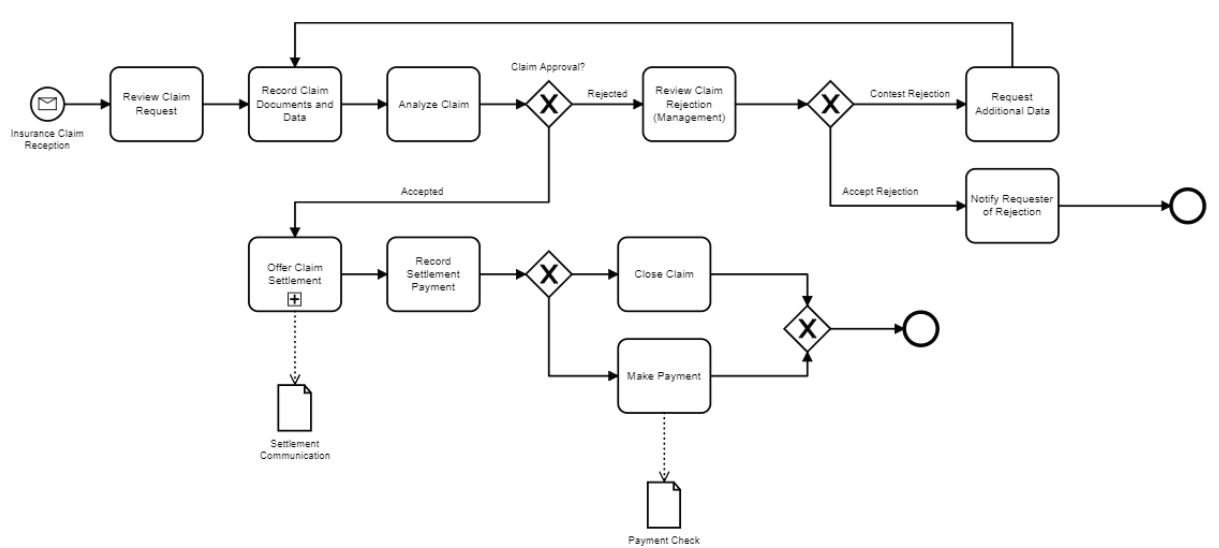

Fig. 6. Example BPMN Insurance Claim Processing model. 
To implement our reality-centric visualization principle, we chose to initially visualize all elements and relations of both ArchiMate and BPMN and allow the user to hide those not desired. As with the death-star-like microservice depictions, while it may initially appear overwhelming, the user is presented with everything that exists in the models, rather than not knowing and then attempting to search for or discover these "accidentally". Moreover, they can navigate to those areas of interest, and planes can be turned on or off. To support fly-through navigation, the left trackpad controls altitude and the right one forward, backward, left, and right movement. Element labels orient themselves to the camera position as one navigates. The labels are semi-transparent, to allow one to know that an element is behind one, but this affects readability negatively. Nevertheless, we find it important to see the structure, and one can navigate to another location to better read a label of interest. Orthogonal layout of connectors was used and connector types are place along the connector in order to avoid collision issues with placement. ArchiMate does not provide bend point information, whereas BPMN does. Inter-model connectors (between BPMN and ArchiMate or between BPMN diagrams) can be placed across models to associate corresponding elements of interest.

We ran into issues with the normalized ArchiMate models missing information that can be used for proper element placement, thus we place elements next to each other when lacking proper positioning and bend-point information. We noticed that other ArchiMate tools also exhibit this issue (see Fig. 7) when they import an exported ArchiMate file (which does not contain the additional information retained by the tools in which the modeling was performed in their proprietary format).

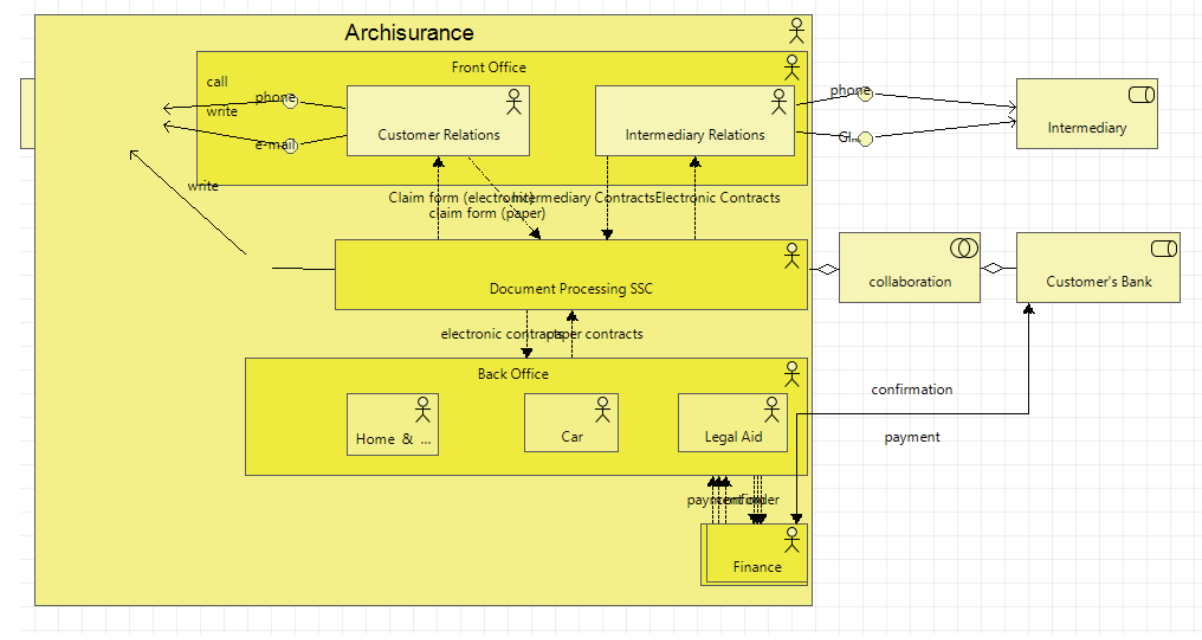

Fig. 7. Unadjusted Modelio import of ArchiSurance Actor Cooperation View (note foreground/background/overlap and containment issues for Finance, HRM, Product Development) 


\section{$5 \quad$ Evaluation}

To evaluate and compare VR-EA with typical alternative 2D ArchiMate tools such as Archi, we use the well-known ArchiSurance case study (Open Group, 2017a) used to illustrate the ArchiMate modeling language (whereby we apply it outside of its TOGAF context). Fig. 8 shows the ArchiSurance model loaded in VR-EA.

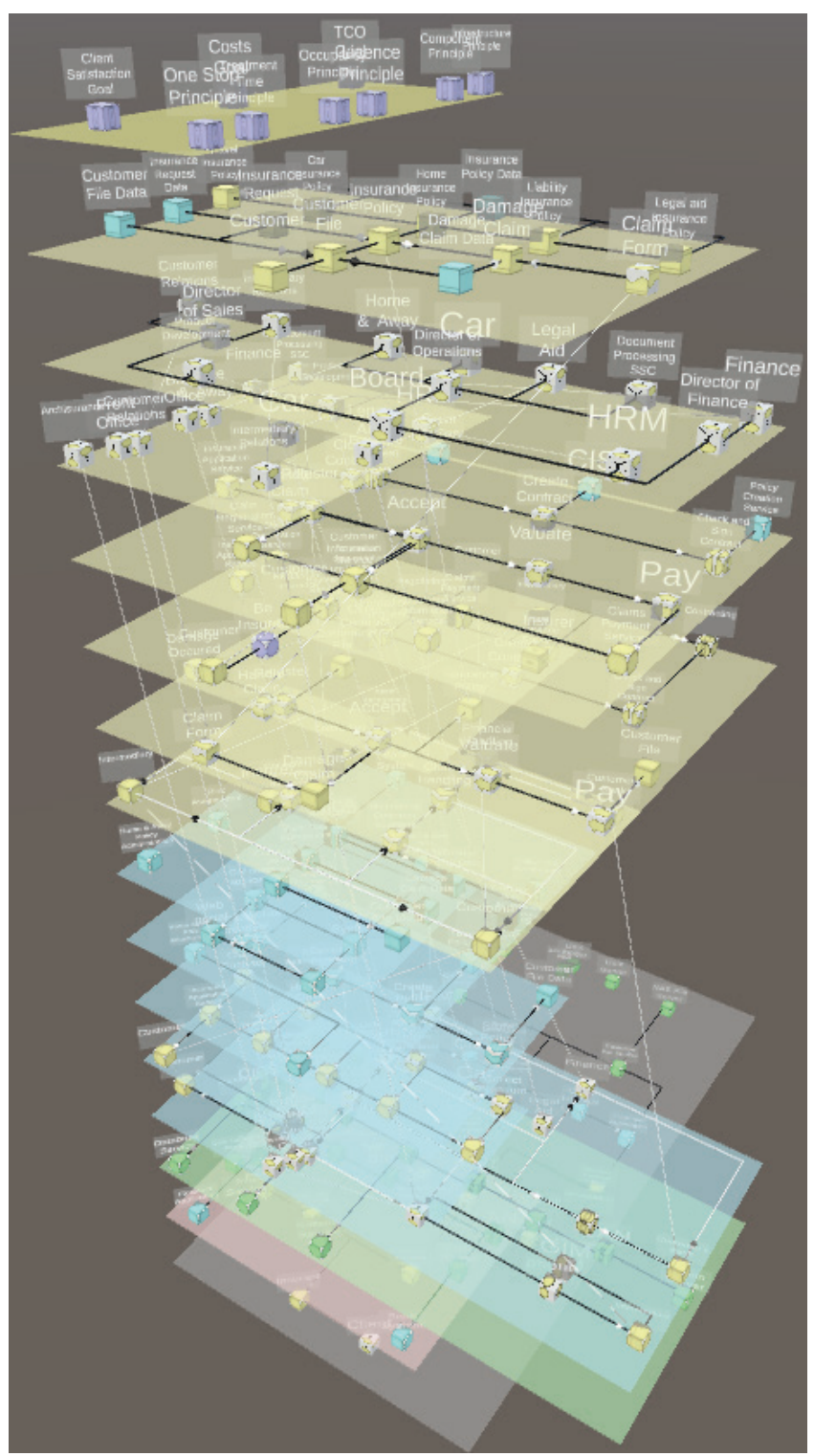

Fig. 8. All ArchiSurance elements and relations visualized in VR-EA 
Based on our reality-centric visualization principle, we intentionally keep the inter-view connections visible to convey the underlying relations and actual complexity (reality) not readily seen with typical 2D rendering of views. This is analogous to the deathstar-like visualizations for microservices referred to in Sec. 1, which we note could also be simplified if desired.

Fig. 9 shows the ArchiSurance Actor Cooperation View in the tool Archi. In contrast, Fig. 10 shows this view in VR-EA with containers shown via blue rectangles. Due to a bug (to be fixed shortly) with our automatic container layout placement implementation, we manually corrected their positioning before taking the screenshots.

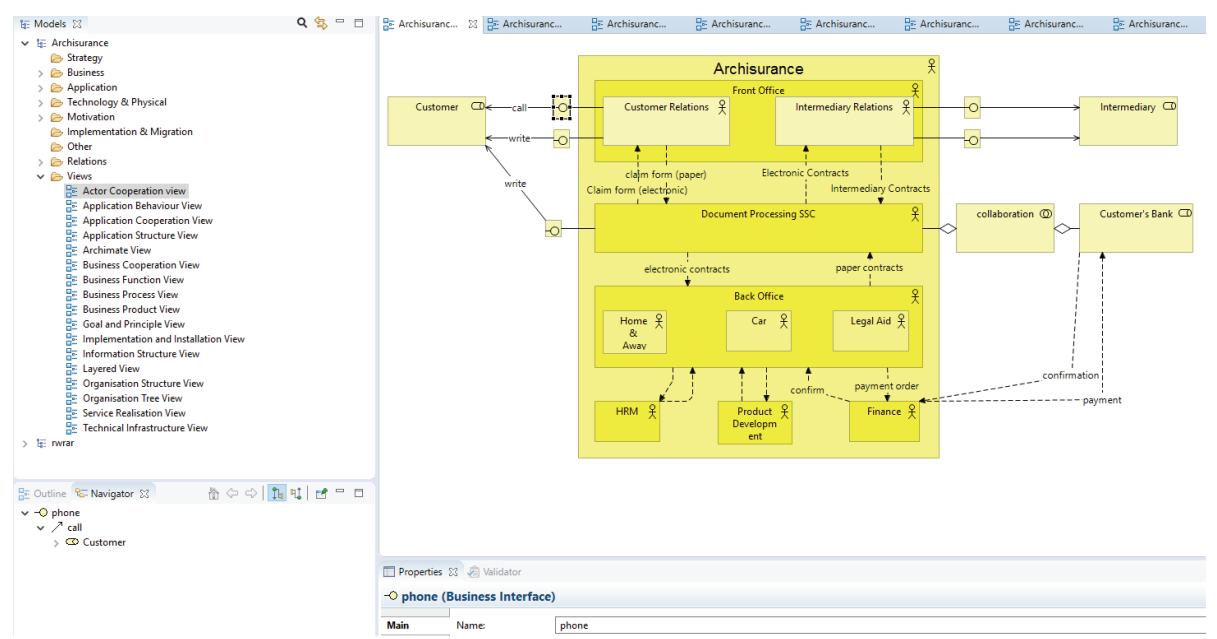

Fig. 9. Screenshot of the ArchiSurance Actor Cooperation View shown in Archi

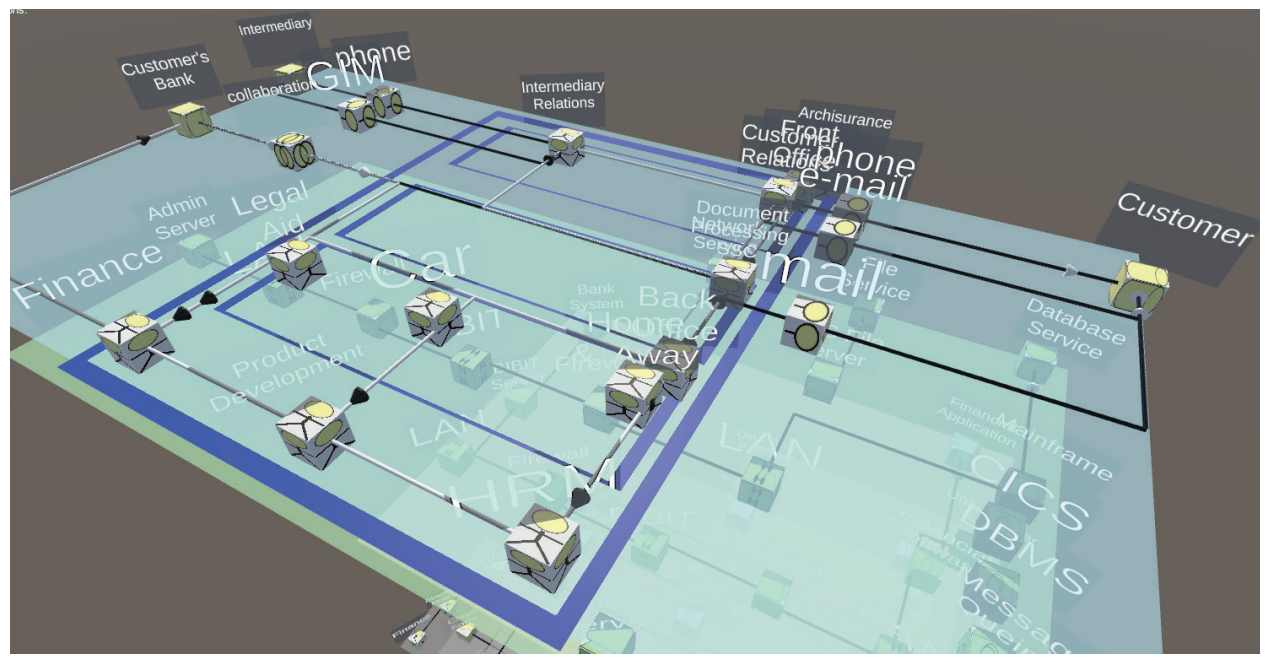

Fig. 10. VR-EA showing the Actor Cooperation View and showing containment as blue boxes 
Fig. 11 shows the ArchiSurance Technical Infrastructure View in Archi and in Fig. 12 in VR-EA.

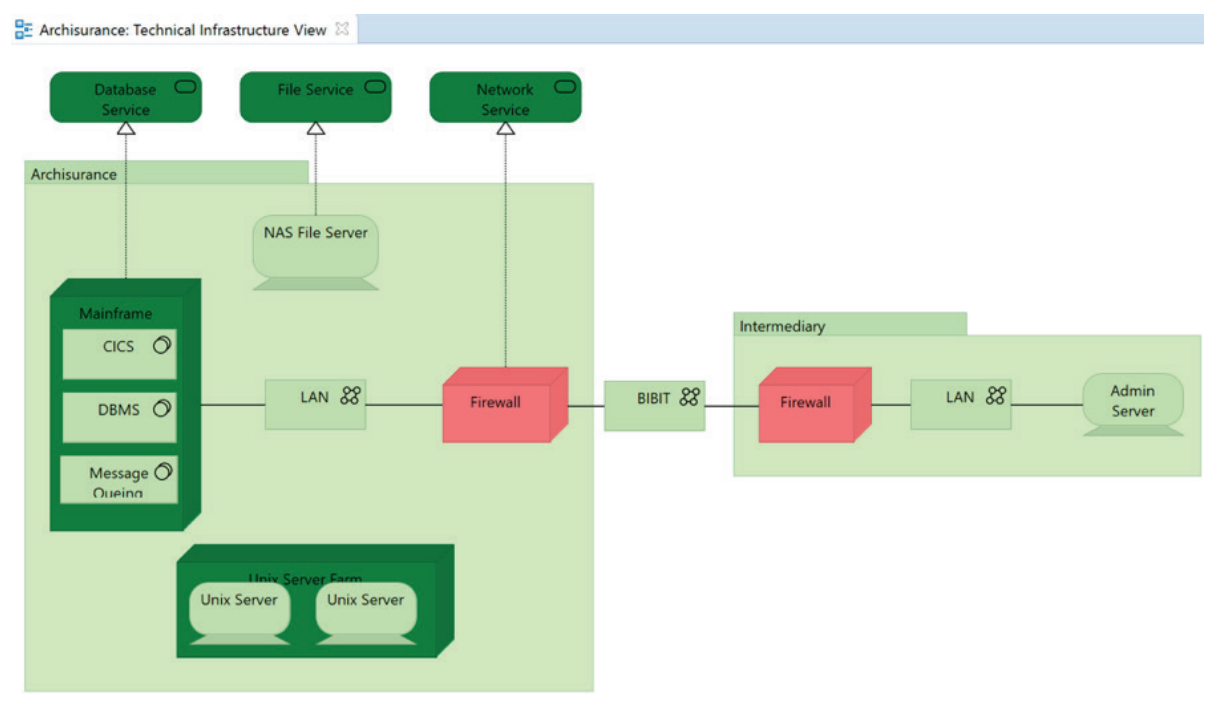

Fig. 11. Screenshot of the ArchiSurance Technical Infrastructure View in Archi

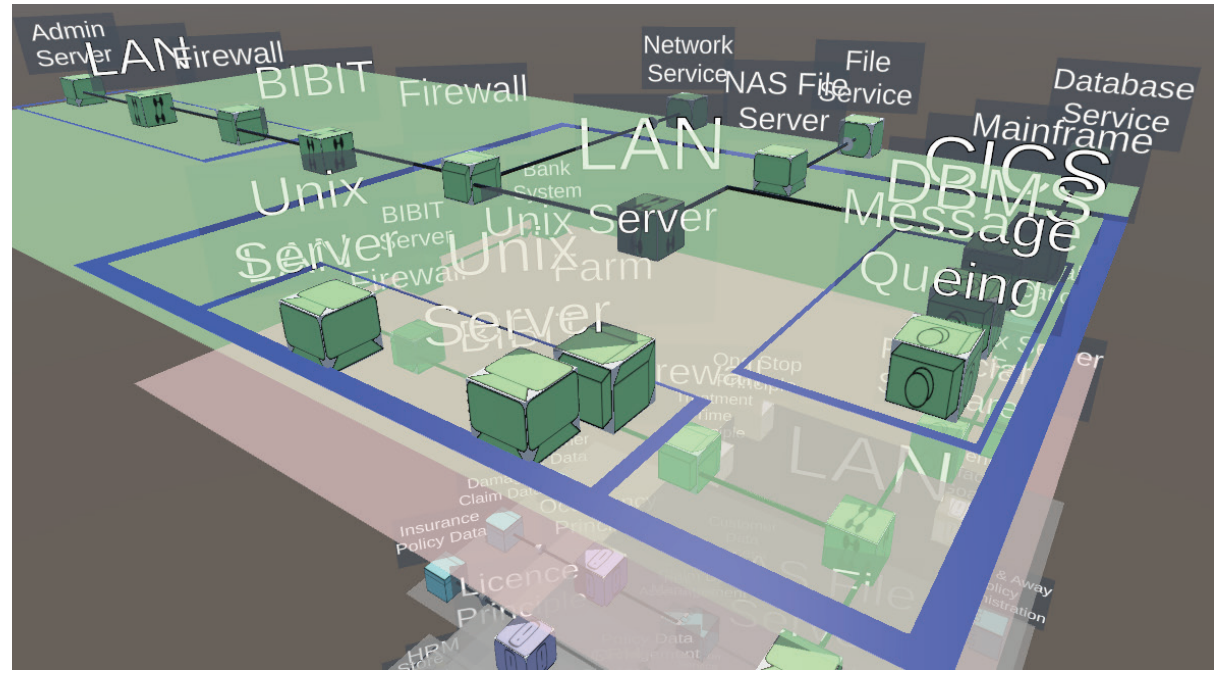

Fig. 12. VR-EA ArchiSurance Technical Infrastructure View (partial) showing containment via blue flat boxes 
Fig. 13 shows the ArchiSurance Business Cooperation View in Archi and in Fig. 14 in VR-EA.

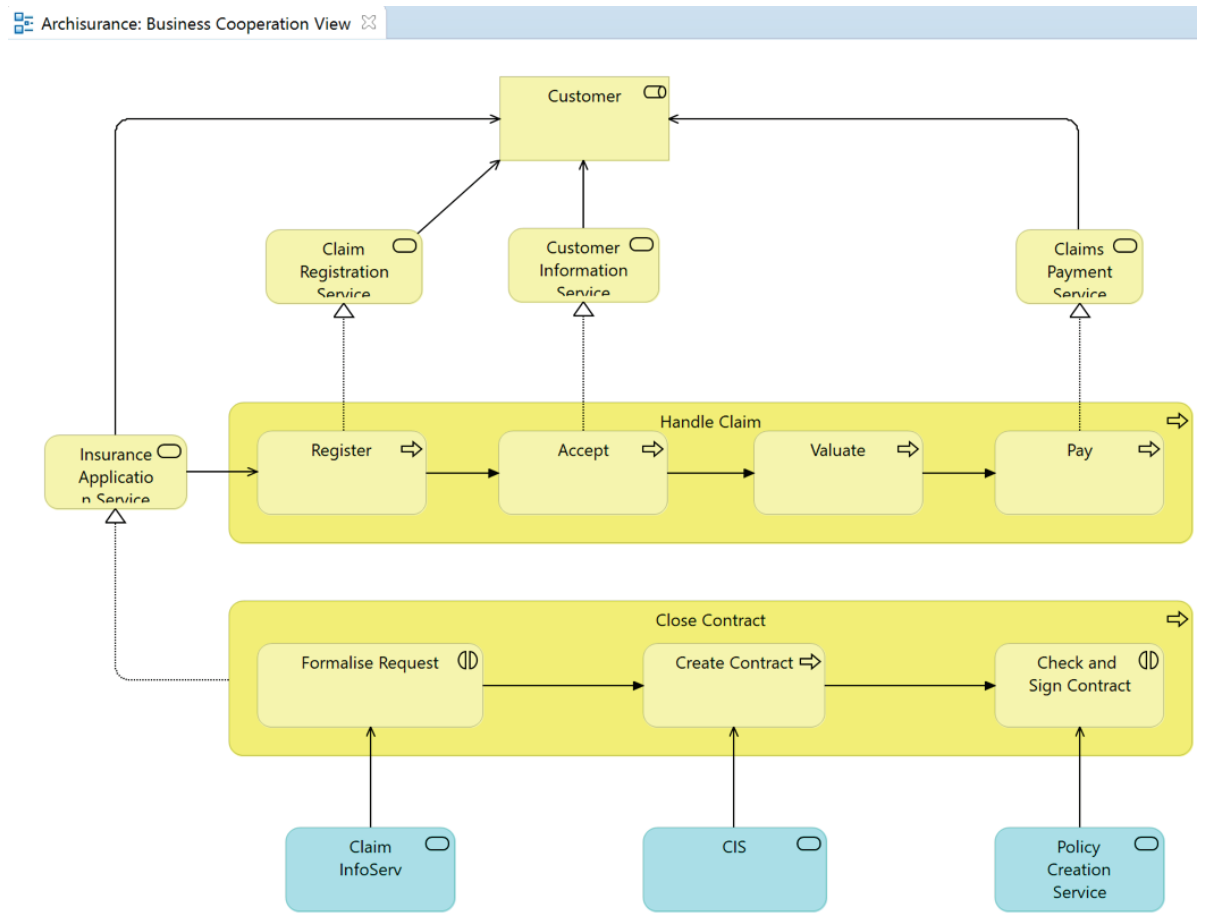

Fig. 13. Screenshot of the ArchiSurance Business Cooperation View in Archi

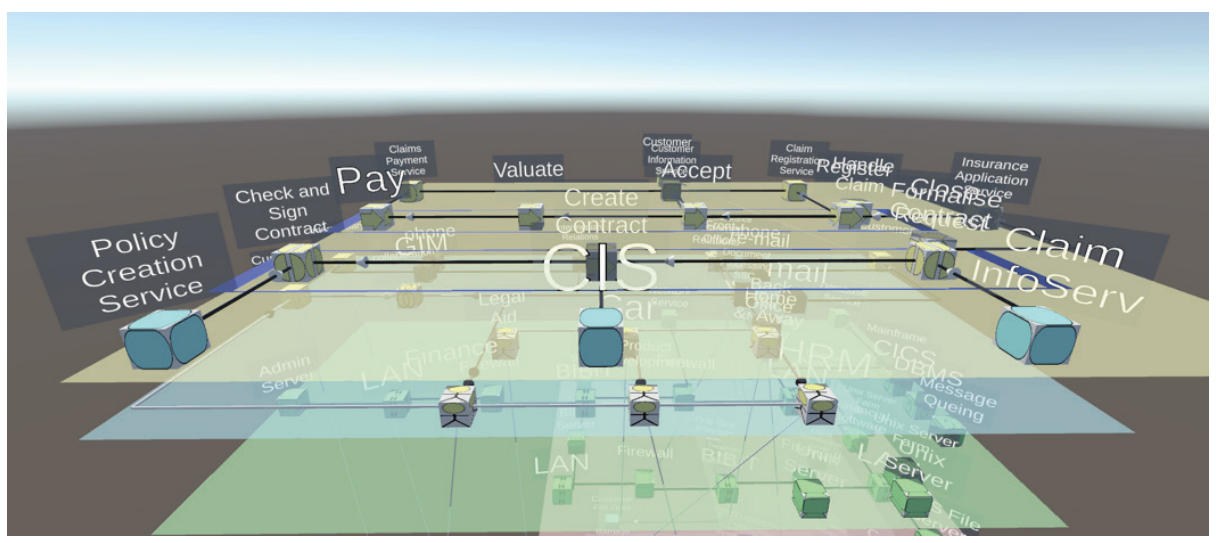

Fig. 14. VR-EA ArchiSurance Business Cooperation View (containers as blue rectangles) 
Fig. 15 shows the ArchiSurance Implementation and Installation View in Archi and in Fig. 16 in VR-EA.

品- Archisurance: Implementation and Installation View ¿3

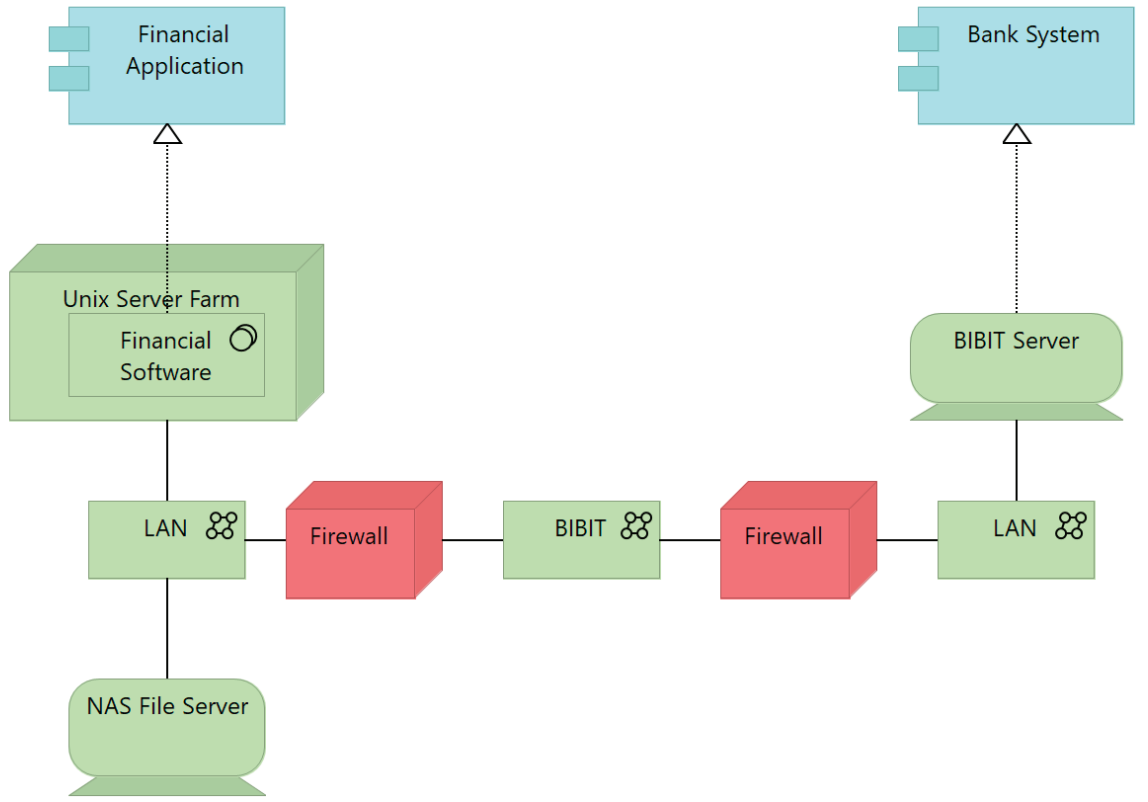

Fig. 15. Screenshot of the ArchiSurance Implementation and Installation View in Archi

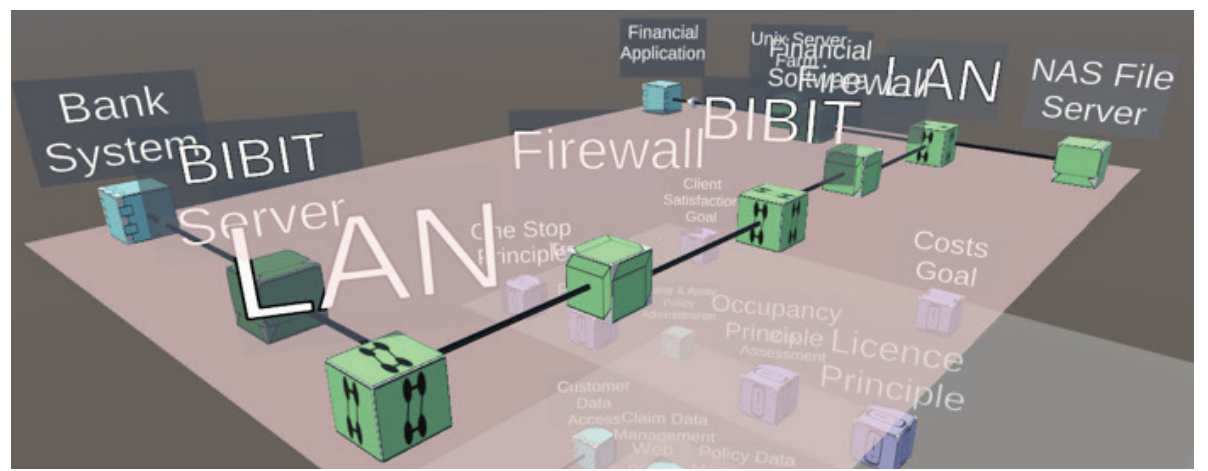

Fig. 16. VR-EA ArchiSurance Implementation and Installation View 
While white cross-view lines indicate where elements reoccur in other views, if a stakeholder wishes to determine where the Customer is directly involved, in VR-EA these are immediately evident by selecting the Customer inter-view connector (shown in red in Fig. 17), enabling one via emphasis to follow the colored connector (red) to all Customer elements in various views.

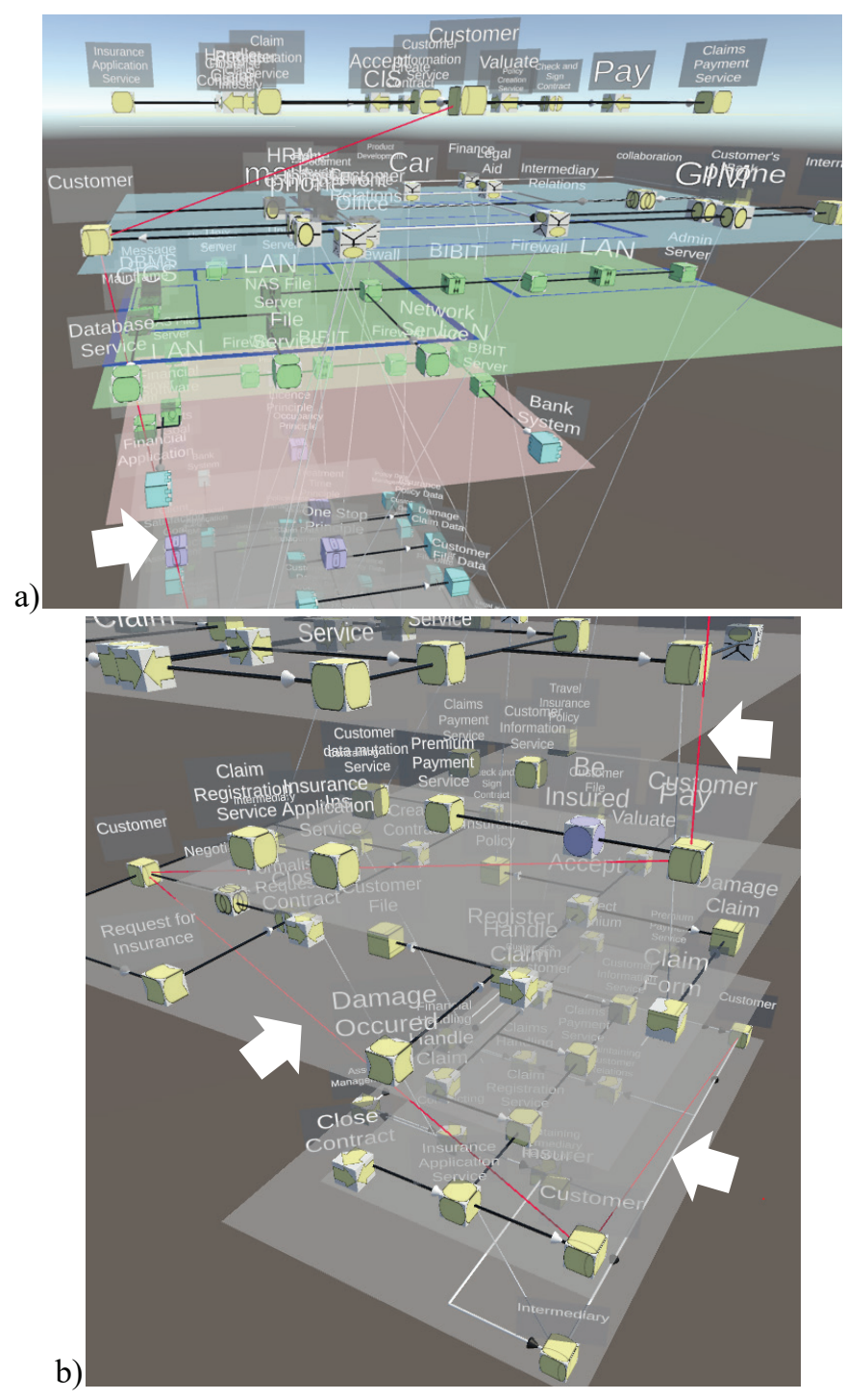

Fig. 17. ArchiSurance Customer inter-view connector selection (colored red) spanning 6 views: a) top perspective with 2 views and b) bottom perspective spanning 4 views (white arrows added to emphasize the red-colored connector) 
In contrast, no ArchiSurance view provides this information directly. As an ArchiMate tool example, Archi offers the ability to generate a view for the Customer as shown in Fig. 18a or by selecting the Customer element and using the Visualizer (Fig. $18 \mathrm{~b}$ ), but the associated views and thus context for these elements are still not clear. In essence, this Customer reality and all the rest of the reality remains hidden in such a tool until one explicitly creates views that show it.

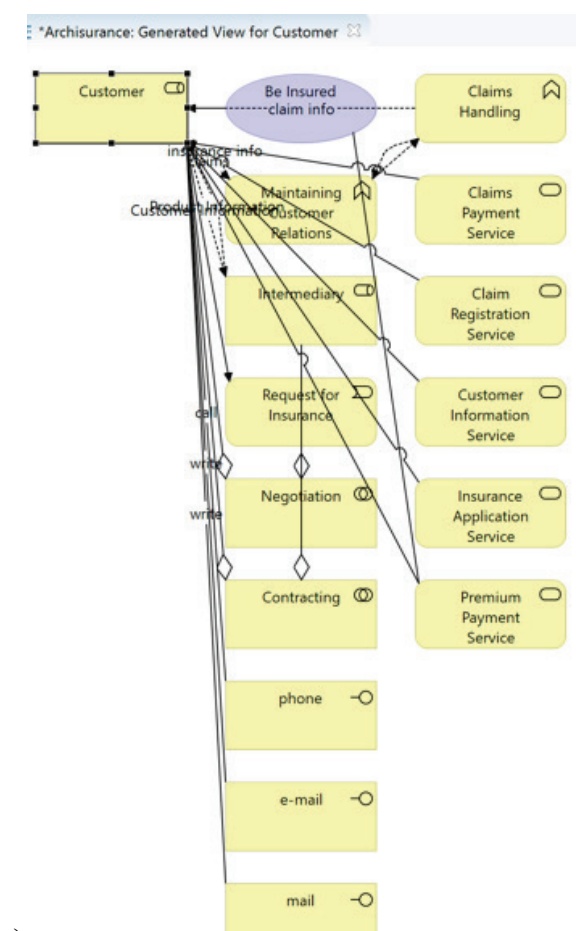

a)

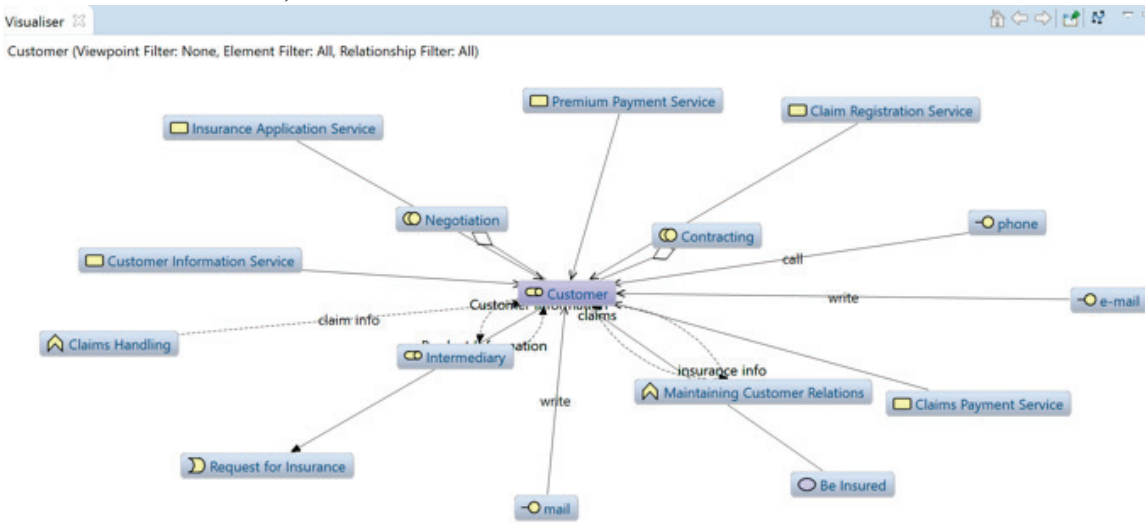

Fig. 18. ArchiSurance screenshots in Archi (a) Generated View for the Customer (top) and (b) Visualiser for the Customer (bottom) 


\section{Conclusion}

With our VR modeling framework VR-MF, we contributed a generalized approach for loading and visualizing different models in VR to create an immersive experience with hypermodels. With VR-EA, we have shown the specific capability to display enterprise architecture models existing in the ArchiMate and BPMN format and addressed the visualization, navigation, and interaction aspects affected by VR. A prototype implementation based on the Unity game engine und using the HTC Vive demonstrated the feasibility of VR-EA. Our evaluation using the ArchiSurance case study compared its capabilities with that of another ArchiMate tool, showing how various views can be portrayed simultaneously and how element interconnectedness is depicted.

While various 2D and 3D data visualization formats are possible based on ArchiMate and BPMN model data, these remain non-immersive. We see a great potential in VR since it can visualize objects (that have symbolic meaning to the users) in a spatial format and allow them to navigate and interact with them in this space. Two dimensional graphical visualization are limited in the ability and area on which they can inter-view interrelations between views, and with the growing IT complexity, while views have their place for simplifying, for certain more complex tasks, such as determining impacts across various views affecting diversified interests of various stakeholders, VR has the advantage of managing to provide an overview of all elements and relations (across all views and diagrams), while still permitting those not of interest to be hidden if desired to reduce complexity. In essence, one can more easily quickly view cross-cutting aspect-like custom $3 \mathrm{D}$ views and navigate these. In our opinion, this can lead to improved EA modeling in the future, but this needs further investigation. Future work will go beyond visualization and navigation and provide new modeling capabilities, offering different layout options, integrating additional interactive and informational capabilities, and will study its usage in practice.

\section{References}

1. Betz, S., Eichhorn, D., Hickl, S., Klink, S., Koschmider, A., Li, Y., Oberweis, A., Trunko, R.: 3D representation of business process models. In: MobIS, pp. 73-87 (2008)

2. Brown, R. A.: Conceptual modelling in $3 \mathrm{D}$ virtual worlds for process communication. In: Proceedings of the Seventh Asia-Pacific Conference on Conceptual Modelling-Volume 110, pp. 25-32. Australian Computer Society, Inc. (2010)

3. Brown, R., Recker, J., West, S.: Using virtual worlds for collaborative business process modeling. Business Process Management Journal, 17(3), 546-564 (2011)

4. $\mathrm{Du}, \mathrm{X} ., \mathrm{Gu}, \mathrm{C}$., Zhu, N.: A survey of business process simulation visualization. In International Conference on Quality, Reliability, Risk, Maintenance, and Safety Engineering (ICQR2MSE), pp. 43-48. IEEE (2012)

5. Effinger, P.: A 3D-navigator for business process models. In: International Conference on Business Process Management, pp. 737-743. Springer, Berlin, Heidelberg (2012) 
6. Forsgren, N., Kim, G., Kersten, N., Humble, J., \& Brown, A.: 2017 State of DevOps report. Puppet + DORA. https://puppet.com/resources/whitepaper/2017-state-of-devopsreport

7. GitHub. (2017) https:/github.com/camunda/camunda-bpm-platform/blob/master/ examples/invoice/src/main/resources/invoice.v2.bpmn

8. Hipp, M., Strauss, A., Michelberger, B., Mutschler, B., Reichert, M.: Enabling a userfriendly visualization of business process models. In International Conference on Business Process Management, pp. 395-407. Springer, Cham (2014)

9. Jarvis, B.: Enterprise Architecture: Understanding the Bigger Picture - A Best Practice Guide for Decision Makers in IT. The UK National Computing Centre, Manchester, UK (2003)

10. Munns, C.: I Love APIs: Microservices at Amazon. (2015) https://www.slideshare.net/apigee/i-love-apis-2015-microservices-at-amazon-54487258

11. Muro, M., Liu, S., Whiton, J., Kulkarni, S.: Digitalization and the American Workforce. Brookings Institution Metropolitan Policy Program (2017). https://www.brookings.edu/wpcontent/uploads/2017/11/mpp_2017nov15_digitalization_full_report.pdf

12. Naranjo, D., Sánchez, M., \& Villalobos, J.: Towards a unified and modular approach for visual analysis of enterprise models. In: 2014 IEEE 18th International Enterprise Distributed Object Computing Conference Workshops and Demonstrations (pp. 77-86). IEEE (2014)

13. Oberhauser, R., Pogolski, C., Matic, A.: VR-BPMN: Visualizing BPMN Models in Virtual Reality. In: International Symposium on Business Modeling and Software Design, pp. 8397. Springer, Cham (2018)

14. OMG: Business Process Model and Notation (BPMN) Version 2.0. OMG (2011)

15. Open Group: ArchiMate 3.0.1 Specification. The Open Group (2017)

16. Open Group: ArchiSurance Case Study, Version 2. The Open Group (2017a)

17. ORYX-Editor: Insurance Claim Processing. BPI https://www.businessprocessincubator.com/content/insurance-claim-processing/

18. Rehring, K., Greulich, M., Bredenfeld, L., \& Ahlemann, F.: Let's Get in Touch-Decision Making about Enterprise Architecture Using 3D Visualization in Augmented Reality. In: Proc. 52nd Hawaii International Conference on System Sciences (HICSS). IEEE (2019)

19. Steuer, J.: Defining virtual reality: Dimensions determining telepresence. Journal of communication, 42(4), 73-93 (1992)

20. van den Berg, M. \& Real IRM Solutions. ArchiMate, BPMN and UML: An approach to harmonizing the notations. Orbus, software, white paper (2012) 